\title{
A Clinical Study of Smell Disorders in COVID-19 Patients in a Tertiary Care Hospital in Pondicherry: A Cross Sectional Study
}

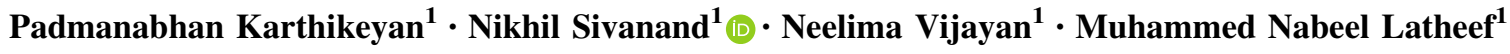

Received: 20 January 2021 / Accepted: 2 March 2021 / Published online: 13 March 2021

(C) Association of Otolaryngologists of India 2021

\begin{abstract}
Anosmia and ageusia are the first and maybe the only symptom in patients affected with COVID-19 especially if the patient is paucisymptomatic. This aim of this study was to determine the demographic details of patients with anosmia, prevalence of anosmia and the time taken for it to resolve in patients who are positive for COVID-19 and took treatment in our hospital. Cross Sectional Study. Patients with real time polymerase chain reaction (RTPCR) positive nasopharyngeal and oropharyngeal swabs, who met the inclusion and exclusion criteria were included in the study. The study group was interviewed through telephonic calls and a questionnaire filled to see the development and regression of their symptoms. Of the study population of 1000, 742 patients had some sort of a smell disturbance. There was a positive correlation between the severity of the disease and history of smoking. The prevalence of smell disturbances among COVID-19 patients in our study was $74.2 \%$. One important finding that we found out was that majority of the smokers had moderate disease. Most of the patients had complete recovery form smell disturbance in the due course of time. The mean time for resolution of smell disturbance was found to be 9.89 days. Anosmia and ageusia can represent the only symptomatology present in patients with COVID-19 and they are completely reversible and hence they can be used as early predictors of infection.

Level of Evidence: Level 2.
\end{abstract}

Nikhil Sivanand

nikhil.sivanand@gmail.com

1 Department of ENT, Mahatma Gandhi Medical College and Research Institute, Sri Balaji Vidyapeeth, Pillaiyarkuppam, Pondicherry 607402, India
Keywords COVID-19 · Coronavirus · Anosmia . Otorhinolaryngology

\section{Introduction}

Several patients with pneumonia of unidentified causes were seen in the Wuhan city of China by the end of 2019, which was later on named as COVID-19 by the World Health Organization. From then on,there was a steady and a very heavy spill over to various parts of the world. Coronavirus disease is an acute respiratory illness caused by a novel human coronavirus (SARS-CoV-2, called COVID-19 virus), which causes higher mortality in people aged $\geq 60$ years and also in people with underlying comorbid conditions such as cardiovascular disease, chronic respiratory disease, diabetes, and cancer. The COVID-19 outbreak was announced as a public health emergency of international concern on January 30, 2020 [1].

The initial symptoms of the disease are fatigue, fever, dry or productive cough, shortness of breath, muscle pain, diarrhea, vomiting, anorexia, headache, sore throat, dizziness, palpitations and chest pain. As more and more cases were reported, the spectrum of symptoms also got expanded and included few symptoms of smell disturbancesand taste disturbances also. As the number of cases reported in medical literature increased, there has been an increment in the number of cases which presents with anosmia or ageusia alone as the presenting complaint. A retrospective study by Gilani et al., in April 2020 reported 8 cases of anosmia of which 5 patients were confirmed cases of COVID-19 [2]. Corona virus is known to cause the common cold like symptoms which is associated with olfactory loss [3]. The initial reports from China did not include anosmia as a presenting symptom of COVID-19. 
A possible association between COVID-19 and anosmia was first reported in a short communication from Italy in the month of July, 2020 and it was a warning symptom especially for the otorhinolaryngologists regarding this possible new manifestation of COVID 19. They reported the presence of anosmia and ageusia as the first and maybe the only symptom in patients affected with COVID-19 especially if the patient is paucisymptomatic [4].

We aimed to determine the demographic details of patients with anosmia, prevalence of anosmiaand the time taken for it to resolve in patients who are positive for COVID-19 andtook treatment in our hospital.

\section{Materials and Methods}

This was a cross sectional study done at Mahatma Gandhi Medical College and Research Institute, Pondicherry, India from a period of August 2020 to December 2020 after obtaining clearance from the Institutional Research Committee of our University. Patients with real time polymerase chain reaction (RTPCR) positive nasopharyngeal and oropharyngeal swabs, (positive for COVID-19) were the study population. The first reported case of COVID-19 in Pondicherry was in March 2020, however in-patient care of positive patients in our hospital was started only in August 2020. The real time PCR of the nasopharyngeal and oropharyngeal swabs for the detection of SARS COVID-19 is done in our centre and worldwide, and they were subjected to only telephonic interviews and therefore no ethical consideration is involved in this study.

Inclusion criteria were patients who are laboratory proven SARS-CoV-2 infectionwith a mild or moderate presentation of COVID-19 according to the severity classification of the Massachusetts General Hospital COVID19 treatmentguidance [5] above 18 years who give informed consent to participate in the study. Exclusion criteria were, patients with a severe form of COVID-19, who did not give consent to take part, subjects with incomplete data, previous anosmia and those who were lost to follow-up (not responding to 2 telephone calls) were excluded.

The study included a convenience sample of SARSCoV2 polymerase chain reaction (PCR) confirmed cases being admitted for care or assessed and discharged during the study period and was optimized to 1000.Patient underwent a telephonic interview on the development of symptoms and then they were serially interviewed at one week intervals for up to 5 weeks or when the symptoms regress, on the progression and regression of symptoms and the time taken for the smell disturbance to resolve was noted. Interview data were anonymously collected and no reward was offered for participation.
Data regarding the demography, history of recent travel,presenting symptoms (fever, myalgia, cough, dyspnea,fatigability, sore throat, anosmia, ageusia, diarrhea, etc.), the severity of the disease, past nasal and paranasal diseases (NPND) and history of smoking were taken fromevery patient who were included in the study (Fig. 1).

\section{Results}

The age of the patients ranged from 18 to 68 years with a mean age of 42.35 years. There was a male preponderance 580 patient $(58 \%)$, with females of $388(38.8 \%)$ and 32 (3.2\%) patients were transgender population. 865 (86.5\%) patients had mild disease as per severity classification of the Massachusetts General Hospital COVID-19 treatment guidance and $135(13.5 \%)$ had moderate symptoms (Tables 1 and 2; Fig. 2).

Most of the cases were without any history of smoking 798 (79.8\%) and majority of the patients with history of smoking were having moderate degree of symptoms (99\%-200 patients) and 2 patients (1\%). Majority of our patients did not have a history of recent travel, 948 patients $(94.8 \%) .625$ patients had a previous history of diagnosed nose an paranasal sinus disease like deviated nasal septum, allergic rhinitis, or sinusitis and 204 patients gave a previous history of nasal surgeries in the past (Table 3; Fig. 3).

We had a total study population of 1000 subjects of which 742 patients had some sort of a smell disturbance, 258 patients did not have any smell disturbance. 163 patients had reduced smell sensation, 123had altered smell sensation and 456 patients had complete loss of smell sensation. Out of the 742 patients with smell disturbances, $652(87.8 \%)$ had complete recovery, 84 (11\%) had partial recovery which means symptoms were better than before and 6 patients $(0.8 \%)$ had no recovery.

Of the study population, 562 patients $(56.2 \%)$ had altered smell sensation as the only presenting complaint, 438 (43.8\%) patients had smell disturbance along with taste abnormalities or other complaints. There was an average resolution time of 7-28 days with a mean of 9.89 days. No death was reported in our study during the study period.

\section{Discussion}

Various theories have been postulated as the causative factor for the development of smell and taste abnormalities in COVID-19. A study by Zhou et al. have reported that COVID-19 uses the same receptor [cellular angiotensinconverting enzyme 2 (ACE 2)] as SARS-CoV [6]. The enzyme found in the tongue. Therefore, it is possible that the COVID-19 causes taste dysfunction in the same way as 
Fig. 1 Questionnaire used for the study

\section{MAHATMA GANDHI MEDICAL COLLEGE AND RESEARCH INSTITUTE [A UNTT OF SRI BALAJI VIDYAPEETH, A DEEMED UNTVERSITY] Pillaiyarkuppam, Puducherry - 607 402 \\ Phone Nos: 0413-2615449 to 2615454 Fax No: 0413-2615457 Ex: 220}

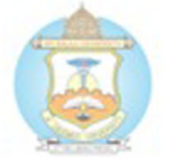

\section{DEPARTMENT OF ENT}

$\underline{\text { Smell Disorders during COVID-19 Pandemic: A Cross Sectional Study }}$

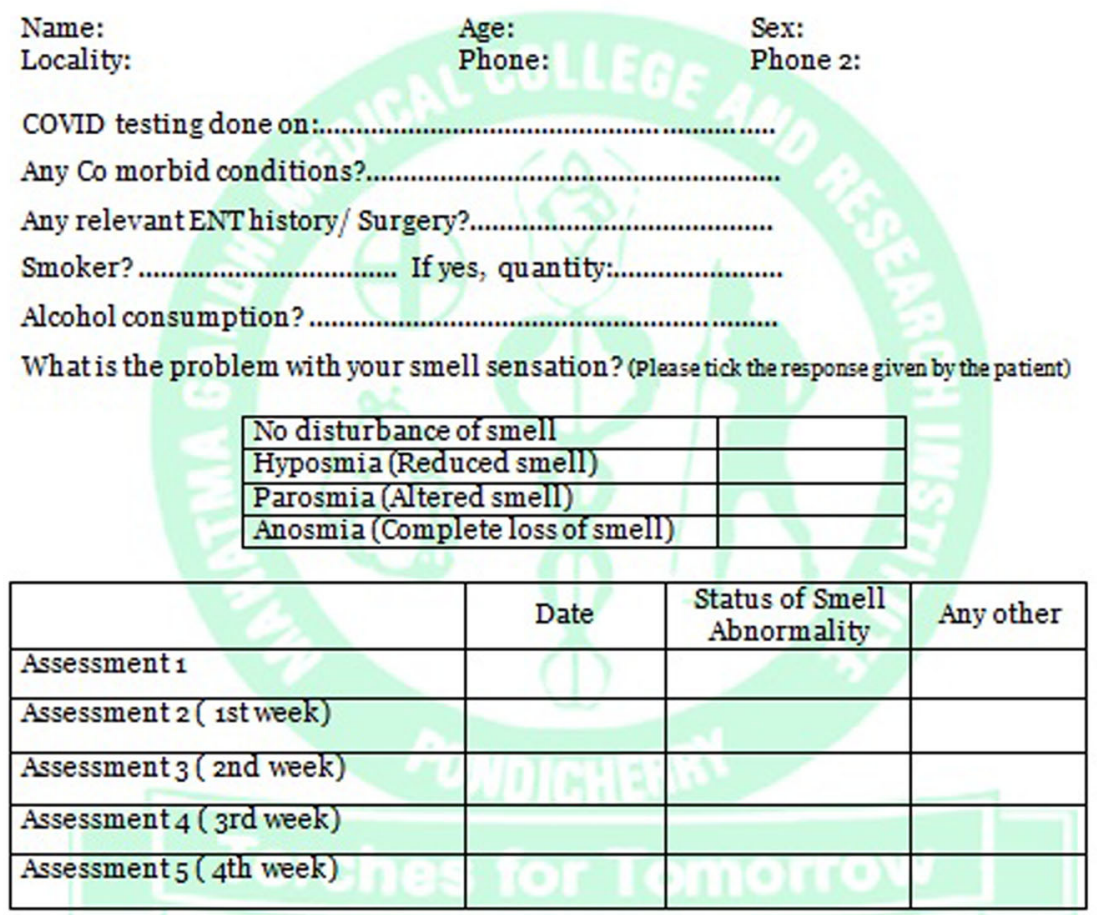

Investigator's Name \& Signature:
Table 1 Incidence of smoking in patients with COVID-19

\begin{tabular}{ll}
\hline $\begin{array}{l}\text { History of } \\
\text { smoking }\end{array}$ & Number of patients \\
\hline Yes & $\begin{array}{c}202 \text { (200 had moderate symptoms and } 2 \text { had mild } \\
\text { symptoms })\end{array}$ \\
No & 798 \\
\hline
\end{tabular}

the ACE2 inhibitors [7]. Secondly, the taste disturbance in the presence of smell abnormality is that both chemosensory senses are closely associated [8]. A study by Brann et al. [9] suggested that the involvement of non-neuronal olfactory epithelium by COVID-19 virus is what causes anosmia and the associated taste dysfunctions. This is the most acceptable mechanism because most of the patients with COVID-19 infection are of the mild severity, outpatient cases, and most of the Smell and Taste abnormalities (STA) resolve within short period of less than 2 weeks [10]. Damaged olfactory cells can regenerate, but they may not regenerate normally and this faulty regeneration is what results in parosmia experienced by most of the patients. However, a larger number of a cohort studies and other meta analyses with long term follow-up and better methods of assessment are needed to assess the exact time and rate of recovery of the STA in confirmed cases of this novel coronavirus infection. 
Table 2 Recent history of travel in patients with COVID-19

\begin{tabular}{lc}
\hline History of travel & Number of patients \\
\hline No & 948 \\
Yes & 52 \\
\hline
\end{tabular}

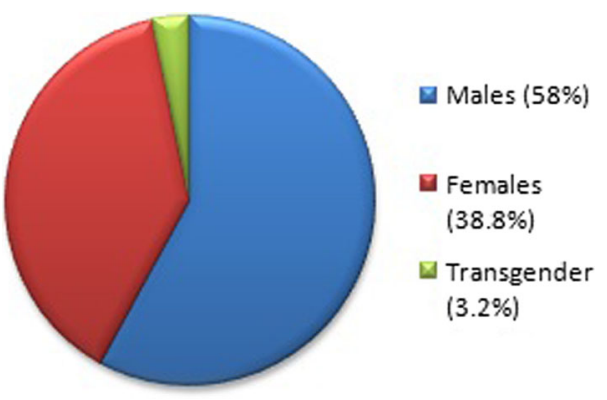

Fig. 2 Sex distribution of patients with COVID-19

Table 3 Incidence of smell disturbances in patients with COVID-19

\begin{tabular}{lc}
\hline Smell Disturbance & Number of patients \\
\hline No disturbance of smell & 258 \\
Hyposmia (Reduced smell) & 163 \\
Parosmia (Altered smell) & 123 \\
Anosmia (Complete loss of smell) & 456 \\
Total & 1000 \\
\hline
\end{tabular}

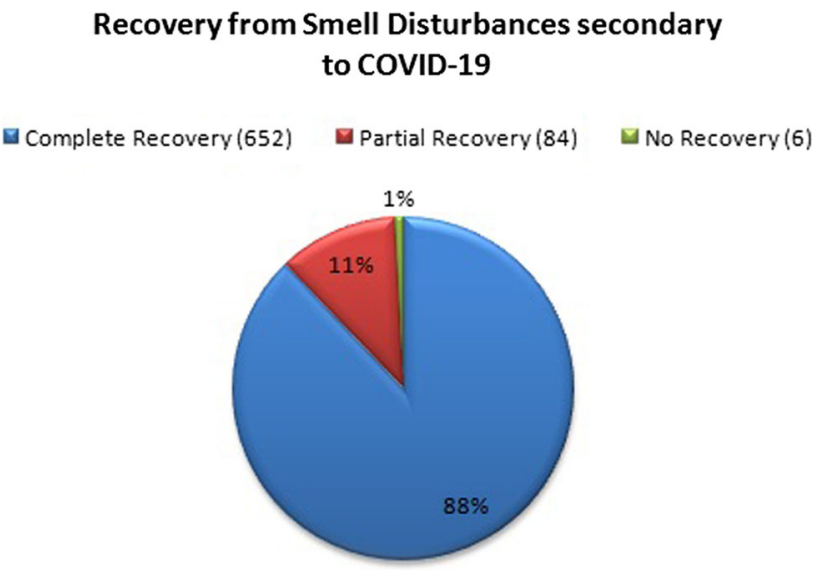

Fig. 3 Recovery from anosmia in patients with COVID-19 and anosmia

Disorders of smell and taste are newly found problems during the pandemic of COVID-19. Smell disturbance alone, smell disturbance with or without taste abnormality or smell disturbance with other COVID symptoms are the usual presenting features noted. Smell and taste abnormalities were the first and the only complaints in $10 \%$ of subjects, $19 \%$ experienced STA before other classical symptoms like fever and cough, as well as $25 \%$ of the children had only STA at the time of the presentation ina study reported by Qiu et al. [11]. Therefore, these disorders are early indicators of COVID-19 disease and can be helpful for screening and infection control. Our study design included a questionnaire for patients about the development of anosmiaalone and the time taken for it to resolve. In a prospective, cross-sectional telephone questionnaire study by Speth et al., who reported that the prevalence rate of olfactory dysfunction was $61.2 \%$ [12]. Another study by Paderno et al., showed that the olfactory dysfunction was seen in $83 \%$ and gustatory dysfunctions i $89 \%$ of the patients [13]. A systematic review of literature by Tong et al. reported the prevalence rate of olfactory dysfunction in COVID- 19 patients as $52.73 \%$ [14]. In a large prospective multicenter case series study by Chary et al., it was found that the rate of STA without nasal complaints was $70 \%$ [15]. This wide variability among various studies might be due to the variation in the ethnicity, differences in sample size, and type of the vast sample (children or adults or both, hospitalized, non-hospitalized or both, with or without nasal symptoms, subjective, objective or both assessment, and severity of the disease).

Our study had a male preponderance of $58 \%$ with an incidence in transgender population in $3.2 \%$. Incidence of COVID-19 has not been reported in literature and the incidence in our study can be because our hospital is a centre for sex reassignment surgery in south India and we have a perennial transgender clinic running in our hospital. This difference between our study and the previous studies might be attributed to the differences in the social and cultural behaviors and geographical locations. All the patients in our study had mild symptoms $(86.5 \%)$ to moderate symptoms (13.5\%) only. This is because our samples were taken from the COVID ward of our hospital and not from the ICU patients or from patients who needed oxygen support.

In the early period of COVID-19 pandemic, recent travel was considered as an important factor for the transmission ofthe disease especially to China or International travel. But as the time progressed and the infection progressed to become a worldwide disease, with community spread, history of recent travel became less and lessimportant. Moreover, the contact with suspicious, asymptomatic or proven COVID-19 cases is an important way of the transmission ofthe infection to healthy people. Our findings did not reveal any significance between recent history of travel and the development of disease.

The effects of smoking and its long term side effects are well established. More smokers were affected and died 
rather than nonsmokers in the MERS-CoV outbreak of 2012 [16]. Smoking has a negative impact on the severity and clinical outcome in patients with the COVID-19 disease [16].In our study, majority of the patients were non smokers $(79.8 \%)$. However, $99 \%$ of the patients with moderate disease were smokers which strengthens the well known fact that smoking causes irreversible lung injury and make them prone to infectious diseases. These finding were contradictory to two similar studies by Speth et al. [12] and by Al-Aniwho reported that $8.8 \%$ of their patients were current smokers and $20 \%$ smokers respectively [17].

Olfactory dysfunction presenting as anosmia or hyposmia are generally one of the first symptoms in COVID-19 disease [12, 18]. A study by Yan et al. [19] reported that a statistically significant association was seen between STA and mild disease and severe disease type was usually not associated with the STA. Therefore, the STA can act as a major positive factor for the prognosis of COVID-19 disease and to segregate patients as mild and moderate disease. Of the study population, 562 patients $(56.2 \%)$ had anosmia as the only presenting complaint, 438 (43.8\%) patients had anosmia along with taste abnormalities or other symptoms like throat pain and dry cough.

In our study, we found that 742 patients in the total study population had smell abnormalities. The prevalence of smell abnormality was found to be $74.2 \%$. Complete recovery was observed in $88 \%$ of the cases, partial recovery was noted in $11 \%$ cases, and lack of recovery in 6 patients $(1 \%)$ after 5 weeks of follow-up. We noted a resolution time range of 7-28 days with a mean of 9.89 days. A study by Vaira et al. noted a spontaneous complete resolution rate of $66 \%$ which was lower in comparison to our study which may be due to the lower study population in their study and it was a self-administered questionnaire [20]. Most of the previous studies have showed a resolution time of about 4-6 days, however the longer resolution time in our study can be attributed to the larger sample size and the objective assessment method [17].

\section{Conclusion}

The COVID-19 pandemic caused by novel human corona virus has made knowledge regarding respiratory and personal hygiene very important [21].The prevalence of smell disturbances among COVID-19 patients in our study was $74.2 \%$. There was a male preponderance with incidence of disease in transgender population also and the mean age group was 42.35 years. Majority of the patients in our study group did not have any travel history and only $20 \%$ of the patients had history of smoking. One important finding that we found out was that majority of the smokers had moderate disease. Most of the patients had complete recovery form smell disturbance in the due course of time. The mean time for resolution of smell disturbance was found to be 9.89 days.

Anosmia and ageusia can represent the only symptomatology present in patients with COVID-19 and they are completely reversible and hence they can be used as early predictors of infection especially for Otorhinolaryngologists.

Funding Not applicable.

\section{Declarations}

Conflict of interest The authors declare that they have no conflict of interest.

\section{References}

1. Cascella M, Rajnik M, Cuomo A, Dulebohn SC, Di Napoli R. Features, evaluation and treatment coronavirus (COVID-19). In: StatPearls [Internet]. Treasure Island (FL): StatPearls Publishing; 2020. Available from: http://www.ncbi.nlm.nih.gov/books/NBK554776/ [Accessed 21 Dec 2020

2. Gilani S, Roditi R, Naraghi M (2020) COVID-19 and anosmia in Tehran. Iran Med Hypotheses 1:109757

3. Hummel T, Whitcroft KL, Andrews P et al (2016) Position paper on olfactory dysfunction. Rhinology 56(1):1-30. https://doi.org/10.4193/Rhin16.248

4. Vaira LA, Salzano G, Deiana G, De Riu G (2020) Anosmia and ageusia: common findings in COVID-19 patients. Laryngoscope 130(7): 1787

5. Massachusetts General Hospital COVID-19 Treatment Guidance2020.

https://www.massgeneral.org/assets/MGH/pdf/news/coronavirus/ covid19_domID_treatmentGuide.pdf. Accessed 21 Mar 2020.

6. Zhou P et al (2020) A pneumonia outbreak associated with a new coronavirus of probable bat origin. Nature 579(7798):270-273

7. Suliburska J, Duda G, Pupek-Musialik D (2012) The influence of hypotensive drugs on the taste sensitivity in patients with primary hypertension. Acta Pol Pharm 69(1):121-127

8. Small DM, Prescott J (2005) Odor/taste integration and the perception of flavor. Exp Brain Res 166(3-4):345-357

9. Brann D, Tsukahara T, Weinreb C, Logan DW, Datta SR (2020) Non-neural expression of SARS-CoV-2 entry genes in the olfactory epithelium suggests mechanisms underlying anosmia in COVID-19 patients. BioRxiv 1:1-10

10. Yan CH, Faraji F, Prajapati DP, Boone CE, DeConde AS (2020) Association of chemosensory dysfunction and Covid-19 in patients presenting with influenza-like symptoms. Int Forum Allergy Rhinol 1:1-6

11. Qiu C et al (2020) Olfactory and gustatory dysfunction as anearly identifier of COVID-19 in adults and children: an international multicenter study. MedRxiv 1:1-6

12. Speth MM, Singer-Cornelius T, Obere M, Gengler I, BrockmeierSJ SAR (2020) Olfactory dysfunction and sinonasal symptomatology in COVID-19: prevalence, severity, timing, and associated characteristics. Otolaryngol Neck Surg 1:1-6 
13. Paderno A et al (2020) Olfactory and gustatory outcomes inCOVID-19: a prospective evaluation in nonhospitalized subjects. Otolaryngol Neck Surg 1:1-10

14. Tong JY, Wong A, Zhu D, Fastenberg JH, Tham T (2020) The prevalence of olfactory and gustatory dysfunction in COVID19patients: a systematic review and meta-analysis. Otolaryngol Neck Surg 1:0194599820926473

15. Chary E et al (2020) Prevalence and recovery from olfactory andgustatory dysfunctions in Covid-19 infection: a prospectivemulticenter study. Am J Rhinol Allergy 1:1945892420930954

16. Kaye R, Chang CWD, Kazahaya K, Brereton J, Denneny JC III (2020) COVID-19 anosmia reporting tool: initial findings. Otolaryngol Neck Surg 1:0194599820922992

17 Al-Ani RM, Acharya D (2020) Prevalence of anosmia and ageusia in patients with COVID-19 at a primary health center, Doha, Qatar. Indian J Otolaryngol Head Neck Surg. https://doi.org/10.1007/s12070-020-02064-9
18. Lechien JR et al (2020) Olfactory and gustatory dysfunctions as aclinical presentation of mild-to-moderate forms of the coronavirusdisease (COVID-19): a multicenter European study. EurArchOto-Rhino-Laryngol 1:1-11

19. Yan CH, Faraji F, Prajapati DP, Boone CE, DeConde AS (2020) Association of chemosensory dysfunction and Covid-19 inpatients presenting with influenza-like symptoms. Int Forum Allergy Rhinol 1:1-6

20. Vaira LA et al (2020) Objective evaluation of anosmia and ageusia in COVID-19 patients: single-center experience on 72 cases. Head Neck 42(6):1252-1258

21 Karthikeyan P, Sivanand N, Vijayan N (2020) Coronavirus disease-19 pandemic: how to flatten the curve? J Basic ClinAppl Health Sci 3(2):90-92

Publisher's Note Springer Nature remains neutral with regard to jurisdictional claims in published maps and institutional affiliations. 